\title{
Use of cultured human epithelium for coverage: A defect of radial forearm free flap donor site
}

\author{
Lorena Gallego $^{1}$, Luis Junquera ${ }^{2}$, Pedro Villarreal ${ }^{3}$, Ignacio Peña ${ }^{4}$, Álvaro Meana ${ }^{5}$ \\ ${ }^{1}$ Resident, Department of Oral and Maxillofacial Surgery. Central University Hospital, Oviedo, Spain \\ ${ }^{2}$ Professor of Oral and Maxillofacial Surgery, University of Oviedo. Spain \\ ${ }^{3}$ Staff Surgeon. Department of Oral and Maxillofacial Surgery. Central University Hospital, Oviedo, Spain \\ ${ }^{4}$ Staff Surgeon. Department of Oral and Maxillofacial Surgery. Central University Hospital, Oviedo, Spain \\ ${ }^{5}$ Director, Tissue Engineering Research Unit, Centro Comunitario de Sangre y Tejidos de Asturias, Spain
}

Correspondence:

School of Dentistry.

University of Oviedo

Catedrático José Serrano $\mathrm{s} / \mathrm{n}$.

33009 Oviedo. Spain

Junquera@uniovi.es

Received: 07/06/2009

Accepted: 02/08/2009
Gallego L, Junquera L, Villarreal P, Peña I, Meana A. Use of cultured human epithelium for coverage: A defect of radial forearm free flap donor site. Med Oral Patol Oral Cir Bucal. 2010 Jan 1;15 (1):e58-60. http://www.medicinaoral.com/medoralfree01/v15i1/medoralv15ilp58.pdf

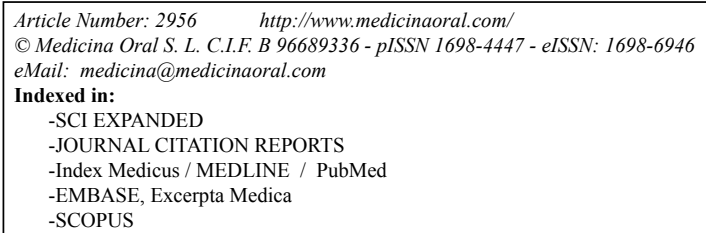

\begin{abstract}
The radial forearm free flap has been popular in many areas of reconstructive surgery. Despite the many attributes of this flap in maxillofacial reconstruction, one of the disadvantages has been the morbidity of the donor site. Allogeneic cultured epidermis has been successfully applied on large second degree burns and on chronic leg ulcers. Autologous human keratinocytes and fibroblast equivalents can be cultured in-vitro from a small skin sample in order to produce a sufficient amount of epithelial autografts to cover the large defects of third-degree burn wounds. Interestingly, transplanted cultured epidermis retains characteristics of the original donor site. We report a case of a patient who underwent skin replacement by cultured epithelial autograft after wound breakdown occurred in the forearm donor site during the early postoperative period. This method could represent an auspicious alternative to conventional grafting methods for forearm free flap reconstruction. To the best of our knowledge, skin replacement by cultured epithelial autografts in this region has not been extensively described in the literature.
\end{abstract}

Key words: Forearm free flap, cultured epithelial autografts, tissue engineering.

\section{Introduction}

Since its introduction by Yang et al. in 1981 (1), the radial forearm free flap has been used extensively for reconstruction of head and neck defects. The radial forearm free flap provides several distinct advantages, as long vascular pedicle, easy technique and good skin quality. The most commonly cited disadvantage is the aesthetic deformity of its donor site when using a split-thickness skin graft. Several techniques have been described to circumvent this problem: secondary healing, full-thick- ness skin graft, use of alloderm, ulnar transposition flaps or tissue expansion (2).

Direct closure would be the method of choice, but is not often possible due to too large defect or insufficient skin laxity. Otherwise, could be associated with delayed wound healing if suture is under tension (3), as the case described.

Autologous cultured human epithelium has been successfully used since 1981 as a permanent coverage of large third-degree burn wounds (4). Interestingly trans- 
planted cultured epidermis retained characteristics of the original donor site (5).

We report a case that underwent skin replacement by cultured epithelial autograft after delayed healing in the donor site of a radial forearm free-flap. To the best of our knowledge, the use of cultured epithelial autografts in this region has not been extensively described in the literature.

\section{Case Report}

A 75-year-old white woman was referred for treatment of a recidivant melanoma of the nose. The patient underwent a fasciocutaneous forearm free flap for reconstruction of a left paranasal facial skin defect after excision of the tumor. The forearm defect was closed primarily. In the early postoperative period, wound breakdown occurred in the donor site, with no spontaneous healing (Fig. 1A). In local anesthesia and under sterile conditions a small skin sample (diameter, $6 \mathrm{~mm}$ ) was taken from behind the ear and the wound was closed with primary suturing. Blood was taken to obtain approximately $50 \mathrm{~mL}$ of blood serum. The wound at the donor site behind the ear was managed in the usual manner and the sutures removed after 7 days. After being washed with antibiotic solution, dermis and subcutaneous tissue were removed from the skin sample under aseptic conditions in the cell (Tissue Engineering Research Unit, Oviedo, Spain).

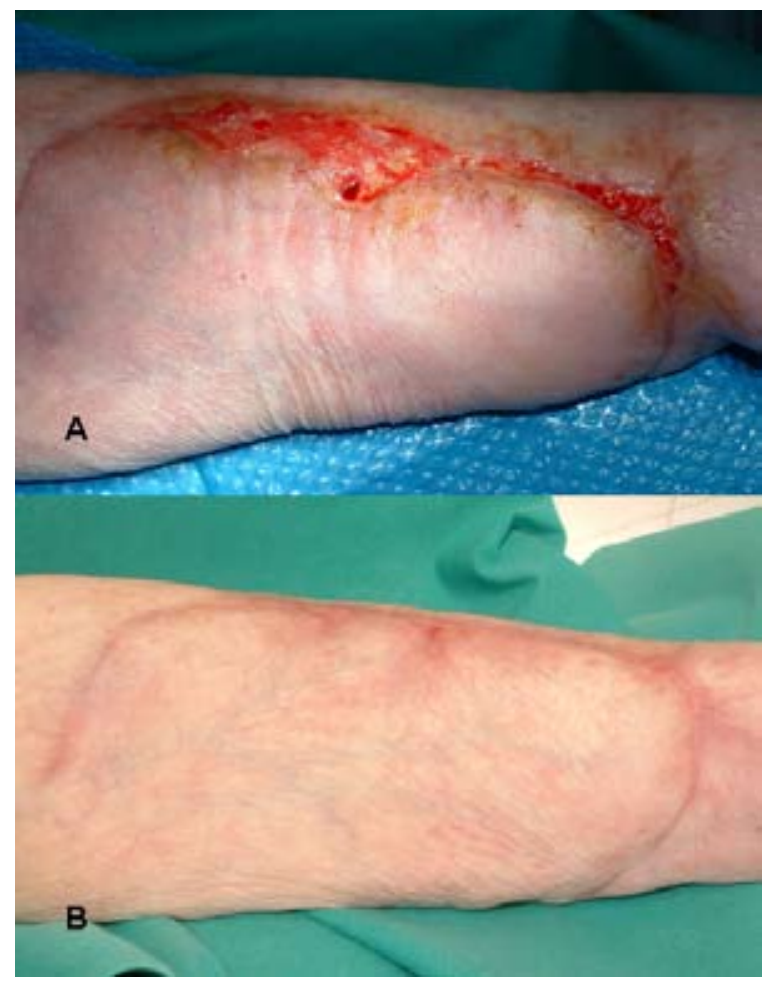

Fig. 1. A.- Aspect of the wound breakdown occurred in the forearm donor site in the early postoperative period. B.- Two weeks after treatment with cultured epithelial autograft. Complete healing with minimum scar contracture and good contour of the forearm is observed.
Cell culture and graft

The keratinocytes were isolated by enzymatic digestion. 2x106 cells obtained were cultivated with 8x106 lethally irradiated 3T3-J2 (Fig. 2), as described by Meana et al. in 1998 (6). Fibroblasts were isolated by enzymatic digestion, and cultured in Dulbecco's modified Eagle medium (DMEM) containing 10\% foetal calf serum (FCS). The primary keratinocytes were seeded in the absence of 3T3 cells, onto a fibrin-fibroblast gel. $1 / 10$ of primary cells in a $75 \mathrm{~cm} 2$ flask was seeded in a $75 \mathrm{~cm} 2$ flask containing the fibrin-fibroblast gel (ratio $1 / 10$ ), and this means $9000 \pm 14,000$ keratinocytes $/ \mathrm{cm} 2$ gel. Keratinocyte growth was followed using an inverted microscope, and when confluence was reached, the gels were manually detached from the culture flask. The complete detachment of the culture from the flask was achieved and confirmed by examining the flask surface using an inverted microscope.

After keratinocyte confluence, cultures on fibrin-fibroblasts gels were transferred to the cleaned and debrided radial forearm flap donor defect in the dressing room under sterile conditions, on an outpatient basis and in local anesthesia. Secure coaptation to wound margin without tension was performed (Fig. 3). Saline-soaked

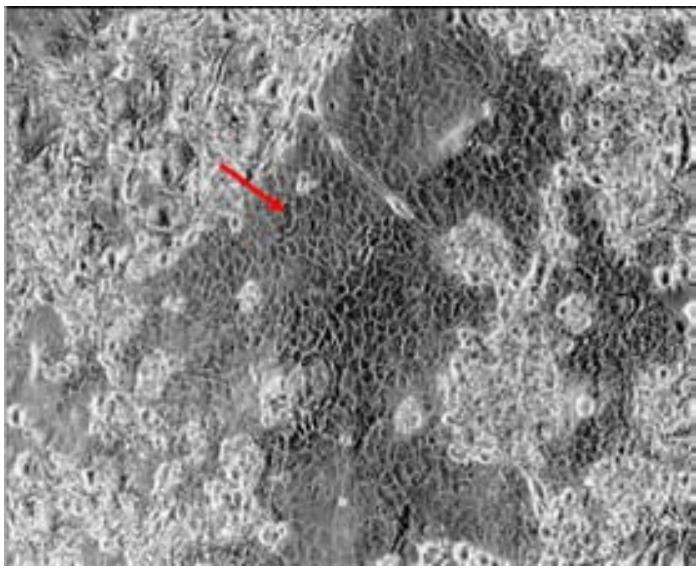

Fig. 2. Microscopic appearance (x100) of a keratinocyte colony (arrow) cultivated with lethally irradiated 3T3.

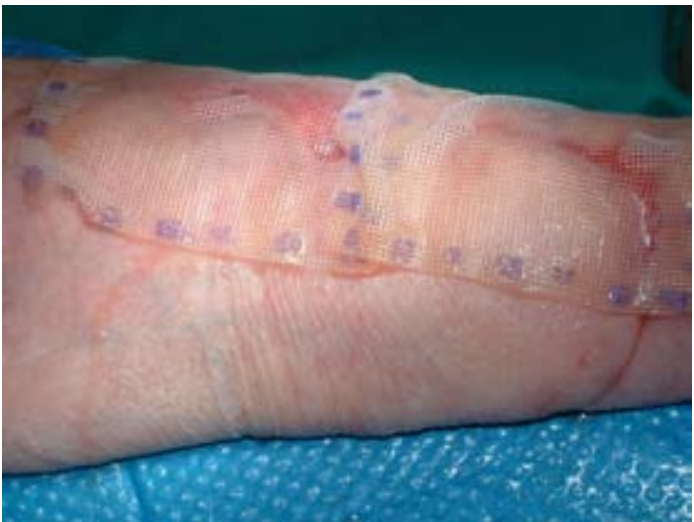

Fig. 3. A cultured epithelial autograft was applied to the radial forearm flap donor defect. 
gauze dressing was applied over the artificial dermis graft, followed by compressive dressing with elastic bandage. The dressing was kept moist with saline solutions to prevent the keratinocytes from drying and to ensure a breakdown of the resorbent matrix. The wrist was maintained in the neutral position by using a volar splint. The dressing was changed first on 5 days after operation, followed by every 2 days. The transplantation sites were monitored at regular intervals. The time for healing was 2 weeks, and healing resulted in visibly normalappearing skin in the donor area (Fig. 1B).

\section{Discussion}

The radial forearm flap has been widely used for the reconstruction of soft-tissue defects in various surgical fields. However, this flap has a clear disadvantage associated with donor site morbidity (7). A variety of techniques have been described to achieve wound healing. Conventionally, the donor site defect was closed with a split-thickness skin graft. However, this method has been associated with significant donor site-related complications such as partial skin-graft loss and flexor tendon exposure. Other techniques include application of a full thickness graft, local skin flaps, tissue expansion and the use of a skin stretching device or artificial dermis (8)

Despite a lot of methods for closing the donor site, none of these suggestions have been universally adopted. When a forearm donor site is small and the surrounding tissue is lax, it may be possible to achieve direct closure of the wound after undermining of the edges. This is probably the best option when is possible without tension.

In 1975 Rheinwald and Green introduced a method for growing epithelial cell sheets in vitro using a feeder layer of irradiated fibroblasts and a serum-containing medium with growth factors. They achieved the first keratinocytes line (XB) starting from a mouse teratoma (9). Two years later the same authors published the obtaining technique by means of in vitro cultivation of human keratinocytes (10). The development in alive of epithelial cells is conditioned by the stimuli fibroblastic, for that the presence in the means of cultivation of mouse fibroblasts lethally radiated (cells 3T3) it allowed the obtaining in cultivation of correctly differentiated epithelium.

We present the use of cultured human epithelium for closing a wound breakdown after direct closure of the donor site. This could be a simple and auspicious alternative to conventional skin grafts to cover donor site defects with good functional and cosmetic result (11). Autologous human keratinocytes and fibroblasts equivalents can be cultured in vitro from a small skin sample to produce sufficiently epithelial autografts to cover the large defects (12). Clinically, the minimization of the morbidity rate in the donor site and the low expenditure of operating time in comparison to conventional methods cannot be overstated (13).

In conclusion, although long-term studies for this procedure need to assess the reproducibility of its results, the available evidence suggests that could be useful for cover donor site defects in these flaps.

\section{References}

1. Yang GF. Free grafting of a lateral brachial skin flap. Zhonghua Wai Ke Za Zhi. 1983;21:272-4.

2. De Witt CA, De Bree R, Verdonck-de Leeuw IM, Quak JJ, Leemans CR. Donor site morbidity of the fasciocutaneous radial forearm flap: what does the patient really bother?. Eur Arch Otorhinolaryngol. 2007;264:929-34.

3. Moazzam A, Gordon DJ. Cross-suturing as an aid to wound closure: a prospective randomised trial using the forearm flap donor site as a model. Br J Plast Surg. 2003;56:695-700.

4. No authors listed. Grafting of burns with cultured epithelium prepared from autologous epidermal cells. Lancet. 1981;1:75-8.

5. De Luca M, Albanese E, Megna M, Cancedda R, Mangiante PE, Cadoni A, et al. Evidence that human oral epithelium reconstituted in vitro and transplanted onto patients with defects in the oral mucosa retains properties of the original donor site. Transplantation. 1990;50:454-9.

6. Meana A, Iglesias J, Del Rio M, Larcher F, Madrigal B, Fresno $\mathrm{MF}$, et al. Large surface of cultured human epithelium obtained on a dermal matrix based on live fibroblast-containing fibrin gels. Burns. 1998;24:621-30.

7. Chen CM, Lin GT, Fu YC, Shieh TY, Huang IY, Shen YS, et al. Complications of free radial forearm flap transfers for head and neck reconstruction. Oral Surg Oral Med Oral Pathol Oral Radiol Endod. 2005;99:671-6.

8. Lee JW, Jang YC, Oh SJ. Use of the artificial dermis for free radial forearm flap donor site. Ann Plast Surg. 2005;55:500-2.

9. Rheinwald JG, Green H. Serial cultivation of strains of human epidermal keratinocytes: the formation of keratinizing colonies from single cells. Cell. 1975;6:331-43.

10. Green H, Kehinde O, Thomas J. Growth of cultured human epidermal cells into multiple epithelia suitable for grafting. Proc Natl Acad Sci U S A. 1979;76:5665-8.

11. Kim DM, Schwerdtner O, Schmidt-Westhausen AM, Kage A, Klein M. Cultured epithelial autografts in the treatment of facial skin defects: clinical outcome. J Oral Maxillofac Surg. 2007;65:439-43.

12. Muhart M, McFalls S, Kirsner RS, Elgart GW, Kerdel F, Sabolinski ML, et al. Behavior of tissue-engineered skin: a comparison of a living skin equivalent, autograft, and occlusive dressing in human donor sites. Arch Dermatol. 1999;135:913-8.

13. Moroi Y, Fujita S, Fukagawa S, Mashino T, Goto T, Masuda T, et al. Clinical evaluation of allogeneic cultured dermal substitutes for intractable skin ulcers after tumor resection. Eur J Dermatol. 2004;14:172-6. 